\title{
Inmunoterapia con pautas agrupadas
}

\section{Immunotherapy with grouped doses}

\section{S. Echechipía, B.E. García, M.T. Aldunate, B. Gómez, E. Lasa, A.I. Tabar}

\section{RESUMEN}

Tras haber sido demostradas la eficacia y la seguridad de la inmunoterapia con extractos alergénicos, en los últimos años se ha hecho patente la necesidad de perfeccionar aquellos aspectos del tratamiento que son mejorables, como es su forma de dosificación. La pauta convencional de la inmunoterapia subcutánea en la fase de incremento de dosis es lenta en alcanzar la dosis considerada eficaz. Por ello se han venido ensaconsayando otras pautas alternativas a la convencional, como las pautas agrupadas, que acorten este periodo de incremento de dosis. Siempre y cuando se garantice la seguridad del tratamiento estas pautas ofrecen las ventajas de reducir el gasto económico y de tiempo, de disminuir la incomodidad del tratamiento y mejorar la adherencia del paciente al mismo y, posiblemente, de alcanzar la eficacia clínica más rápidamente. Sin embargo, no es fácil determinar la pauta de adminismán tracion idónea (mas reacciones adversas) y en este artículo se revisan los problemas existentes a la hora de diseñar estas pauta agrupadas. Por último, se presentan los resultados de un estudio comparativo entre la pauta convencional y una pauta agrupada, de diseño doble ciego, realizado por nosotros, en el que se demuestra que la pauta agrupada es más rápida en conseguir la eficacia clínica deseada, adelanta en el tiempo la disminución de la sensibilidad cutánea al al sensibilidad cut os parametros inmunológicos, todo ello con una frecuencia de reacciones adversas baja y similar a la registrada con la pauta convencional.

Palabras Clave. Inmunoterapia con extractos alergénicos. Pautas. Eficacia. Seguridad.

\section{ABSTRACT}

Once the efficacy and safety of immunotherapy with allergen extracts has been shown, recently it has become evident the need for perfecting those aspect of the treatment that can be improved, such as its dosage form. The conventional dosage of subcutaneous immunotherapy in the phase of dose increase is slow in reaching an efficient level. For this reason other alternative dosages to the conventional reason other alternative one have been tried out, such as grouped dosages, which shorten this period of dose increase. On condition that the safety of the treatment is guaranteed, these doses offer the advantages of reducing the economic cost and the time involved, of reducing the discomfort of the treatment and of improving the patient's adherence to the treatment and possibly of reaching clinical efficacy more rapidly. Nonetheless, it is not easy to determine the suitable ente dosage of administration (the shortest and with the least number of adverse reactions) and this article reviews the existing problems when it comes to designing these grouped doses. Finally, we present the results of a comparative study between the conventional dose and a grouped dose, with a double blind design, carried out by us, which shows that the grouped dose is quicker in achieving the desired clinical efficacy, shortens the times of reduction of cutaneous sensitivity to the allergen and of cutaneous sef sitivity to the allergen a modicication of the immunological parameters, all with a low requency of adverse reactions that is similar to that registered with the conventional dosage.

Key words. Immunotherapy with allergen extracts. Dosage forms. Efficacy. Safety.

An. sis. sanit. Navar. 2003; 26 (Supl. 2): 119-127.

Sección de Alergología. Hospital Virgen del Camino Pamplona.

Este trabajo ha sido parcialmente subvencionado con una Ayuda del Departamento de Salud del Gobierno de Navarra (Resolución 1286/1996).

\section{Correspondencia:}

Susana Echechipía Madoz

Sección de Alergología. Hospital Virgen de Camino

CS Conde Oliveto

Pza. Paz s/n

31002 Pamplona

Tfno: 948429308

Fax: 948429271 


\section{INTRODUCCIÓN}

En la actualidad la inmunoterapia subcutánea con extractos alergénicos es un tratamiento de eficacia reconocida tanto en la anafilaxia por veneno de himenópteros como en las enfermedades alérgicas respiratorias $^{14}$. La pauta convencional de administración del tratamiento es segura ya que ocasiona un reducido número de reacciones adversas ${ }^{5,6}$, pero ha sido establecida arbitrariamente sin que se haya demostrado que sea la pauta óptima de administración. Esta forma de dosificación convencional es lenta en la fase de aumento progresivo de dosis porque requiere de 12 a 16 inyecciones una vez por semana hasta alcanzar la dosis de mantenimiento o dosis eficaz. Por lo tanto supone un gran esfuerzo tanto por parte del sistema sanitario como por parte del paciente, con un gran consumo económico y de tiempo. Esto puede ocasionar un inadecuado cumplimiento del tratamiento por parte del paciente, o bien el abandono del mismo debido a su incomodidad. Así, el intentar disminuir el tiempo necesario para llegar a la dosis de mantenimiento puede mejorar la adherencia del paciente a la inmunoterapia. En el caso de los pacientes alérgicos a venenos de himenópteros, la necesidad de conseguir la eficacia clínica en el menor tiempo posible impulsó la investigación de pautas más rápidas que la convencional en la fase de inicio, ya que son estos pacientes los que presentan riesgo de anafilaxia fatal. Con la experiencia obtenida de estas pautas con venenos, se han diseñado pautas alternativas de administración de la inmunoterapia con otros extractos alergénicos como son ácaros ${ }^{7-10}$, pólenes ${ }^{11-15}$ y hongos ${ }^{16,17}$ El objetivo es acortar el tiempo necesario para alcanzar la dosis de mantenimiento y de este modo reducir el gasto económico y de tiempo, disminuir la incomodidad del tratamiento y mejorar la adherencia al mismo, y posiblemente alcanzar la eficacia clínica con mayor rapidez.

\section{DISEÑO DE PAUTAS AGRUPADAS}

Las pautas agrupadas consisten en la administración de varias dosis crecientes del extracto alergénico en un mismo día separando estos grupos de inyecciones por un intervalo de tiempo variable, habitualmente de una semana, de forma que se reduce el tiempo necesario para alcanzar la dosis eficaz respecto a la pauta convencional. Estas pautas agrupadas ofrecen una mejor relación beneficio-riesgo que otras pautas más rápidas que producen mayor número de reacciones adversas en pacientes en los que no existe una urgencia vital en alcanzar la dosis de mantenimiento, como ocurre en los pacientes con rinitis y asma bronquial.

A la hora de diseñar una pauta agrupada debemos definir 4 cuestiones fundamentales: dosis inicial, magnitud del incremento de dosis, intervalo de tiempo entre grupos de inyecciones y tipo de extracto (acuoso o depot).

Se ha demostrado que dosis iniciales superiores a las administradas en las pautas convencionales son bien toleradas. En un estudio comparativo de la tolerancia de 4 diferentes pautas agrupadas de inmunoterapia con un extracto depot de Alternaria no se registraron reacciones adversas sistémicas ni con las pautas que administraban dosis iniciales bajas ni con aquéllas con dosis iniciales 10 veces superiores ${ }^{17}$. La eliminación de estas dosis bajas de la pauta permite disminuir el número de visitas.

Es difícil establecer qué incremento de dosis es más seguro. Si aumentamos mucho la dosis de un grupo de inyecciones a otro corremos el riesgo de empeorar la tolerancia, pero a la vez no debemos administrar una dosis diaria acumulada demasiado alta ya que también aumenta el riesgo de reacciones adversas. Así la administración de una pauta agrupada de inmunoterapia con extracto acuoso de pólenes en 3 visitas con 5 inyecciones en cada una produjo reacciones sistémicas en un $65 \%$ de pacientes, sobre todo con las dosis más altas $^{14}$. Esta pauta se modificó espaciando las dosis correspondientes al vial de más concentración, con una dosis total acumulada mucho menor y la tolerancia fue excelente, sin registrarse reacciones sistémicas $^{18}$. Con una pauta agrupada en 7 visitas semanales en que se administró un extracto acuoso de ácaros alcanzándose una dosis acumulada diaria muy alta se produjeron reacciones sistémicas en un 33\% 
pacientes ${ }^{10}$. Por el contrario, con un extracto similar pero depot, en una pauta más rápida de 4 visitas frente a 6 y una dosis acumulada 3,5 veces inferior se registraron reacciones sistémicas en un $7,5 \%$ pacientes ${ }^{8}$. Por lo tanto, debemos encontrar un equilibrio en el aumento de dosis para que la dosis acumulada diaria no sea demasiado alta pero a la vez no existan demasiadas diferencias entre la dosis de un grupo de inyecciones y el siguiente.

En cuanto al intervalo de tiempo entre grupos de inyecciones, para poderlo establecer necesitaríamos conocer la farmacocinética y farmacodinámica de los extractos alergénicos así como el periodo de latencia entre su administración y su efecto. Hoy por hoy desconocemos estos aspectos por lo que el intervalo se establece de forma totalmente arbitraria, y en la mayoría de los estudios revisados es semanal, de forma análoga a como se administra la pauta convencional.

Por último en cuanto al extracto a administrar, si bien inicialmente los estudios de pautas agrupadas utilizaban extractos acuosos, en la actualidad la tendencia es a administrar extractos depot, por su mayor estabilidad y porque se ha demostrado su mejor tolerancia. Utilizando pautas agrupadas similares de extractos de pólenes, se produjeron reacciones sistémicas en un $6 \%$ pacientes $(0,6 \%$ dosis $)$ que recibieron el extracto acuoso ${ }^{11} \mathrm{y}$ en un $0,7 \%$ de pacientes $(0,07 \%$ dosis $)$ que recibieron el extracto depot ${ }^{19}$. En una revisión de la tolerancia de 3 diferentes pautas agrupadas de 5 a 7 semanas de duración en que se administraban extractos acuosos en unos casos y depot en otros a 657 pacientes, la mejor tolerancia fue la obtenida con la pauta de 7 semanas de duración utilizando extractos depot ${ }^{9}$.

En la actualidad existe una clara tendencia a utilizar extractos depot por los diferentes grupos investigadores por ser mejor tolerados que los extractos acuosos.

\section{TOLERANCIA DE LAS PAUTAS AGRUPADAS}

El diseño de las pautas agrupadas se ha ido perfeccionando con el tiempo de forma que las que actualmente se vienen utilizan- do no producen la elevada frecuencia de reacciones adversas que tenía lugar con las ensayadas hace 10 años.

Uno de los estudios de tolerancia de extractos depot de pólenes más recientes y en el que hemos participado ha sido realizado en nuestro país. Se trata de un trabajo multicéntrico, abierto, prospectivo y longitudinal en el que fueron incluidos 306 pacientes de 5 a 50 años con rinoconjuntivitis y/o asma por pólenes de gramíneas u olivo. Se siguió una pauta de inicio de 4 visitas con 2 inyecciones en cada visita. La dosis inicial era 10 veces superior a la correspondiente en la pauta convencional. Se registraron reacciones sistémicas en $9,5 \%$ pacientes, leves en su mayoría, y locales en $7 \%$. Al analizar estas reacciones su aparición no estaba en relación con que el extracto administrado fuera de gramíneas u olivo, ni con el diagnóstico del paciente (rinitis o asma), a excepción de las reacciones sistémicas inmediatas que eran más frecuentes en asmáticos. La mayoría de reacciones ocurrieron en el tercer grupo de inyecciones al iniciar el vial de máxima concentración, siendo sobre todo reacciones tardías.

Con ácaros, nuestro grupo diseñó en el año 92 una pauta agrupada de 6 semanas de duración. Realizamos un ensayo piloto para comparar la eficacia y la tolerancia de esta pauta, administrada a 29 pacientes, con la de la pauta convencional, que fue administrada a 15 pacientes. Sólo se registró una reacción sistémica inmediata leve en un paciente que recibió la pauta agrupada $(3,45 \%$ pacientes y $0,1 \%$ dosis administradas) y no hubo diferencias entre la pauta agrupada y la convencional en cuanto a tolerancia ${ }^{20}$

\section{EFICACIA DE LAS PAUTAS AGRUPADAS}

La mayoría de estudios realizados con pautas agrupadas se centran en determinar su tolerancia; muy pocos evalúan parámetros de eficacia y menos aún comparan la eficacia de las pautas agrupadas con la de las convencionales. Ninguno de los trabajos realizados con pautas agrupadas revisados hasta el momento ha valorado $s$ la mayor rapidez en alcanzar la dosis máxi- 
ma supone que la mejoría clínica aparece más precozmente. Partiendo de la experiencia previa ${ }^{20}$, diseñamos un estudio cuyo objetivo ha sido comparar la eficacia y la seguridad de una pauta de inmunoterapia subcutánea con extracto depot de Dermatophagoides pteronyssinus agrupada en 6 semanas en la fase de incremento de dosis con las de la pauta convencional de 12 semanas de duración. Nosotros en este trabajo también hemos pretendido determinar si realmente esta pauta agrupada adelanta en el tiempo la eficacia clínica del tratamiento con respecto a la pauta convencional habitualmente utilizada, lo que supondría una ventaja adicional de la pauta agrupada.

Se trata de un ensayo clínico en fase IV, prospectivo, aleatorizado, de diseño doble ciego controlado con placebo, enmascarando la pauta de administración del tratamiento en la fase de inicio, de dos años de duración.
Determinamos el tamaño de muestra necesario para comparar la seguridad de ambas pautas, agrupada y convencional, basándonos en los datos de reacciones adversas obtenidos en el ensayo previo ${ }^{20} \mathrm{y}$ calculamos que debíamos incluir a todos los pacientes diagnosticados de rinitis y/o asma bronquial leve-moderada causada por $D$. pteronyssinus en nuestra Sección de Alergología a lo largo de un año que tuvieran indicación de inmunoterapia (IT). A todos ellos se les propuso participar en el estudio.

Para enmascarar las pautas de tratamiento se administró a todos los pacientes el mismo número de dosis, unas de extracto alergénico y otras de placebo, según correspondiera a la pauta que a ese paciente se le hubiera asignado. Los esquemas de administración de ambas pautas de inicio se representan en las tablas 1 y 2. Las dosis fueron preparadas semanalmente en el Servicio de Farmacia

Tabla 1. Pauta agrupada

\begin{tabular}{|c|c|c|c|c|}
\hline SEMANA & VIAL & $\begin{array}{l}\text { VOL. } \\
\text { (mI) }\end{array}$ & $\begin{array}{l}\text { DOSIS } \\
\text { (U.B.) } \\
\end{array}$ & $\begin{array}{c}\text { DOSISA CUMULADA } \\
\text { (U.B.) }\end{array}$ \\
\hline 1 & 1 & $\begin{array}{l}0,2 \\
0,4 \\
0,6 \\
0,8\end{array}$ & $\begin{array}{l}0,02 \\
0,04 \\
0,06 \\
0,08\end{array}$ & 0,2 \\
\hline 2 & 2 & $\begin{array}{l}0,1 \\
0,2 \\
0,4\end{array}$ & $\begin{array}{l}0,1 \\
0,1 \\
0,4\end{array}$ & 0,9 \\
\hline 3 & & $\begin{array}{l}0,6 \\
0,8\end{array}$ & $\begin{array}{l}0,6 \\
0,8\end{array}$ & 2,3 \\
\hline 4 & 3 & $\begin{array}{c}0,1+0,7 \mathrm{P} \\
0,2\end{array}$ & $\begin{array}{l}1 \\
2\end{array}$ & 5,3 \\
\hline 5 & & $\begin{array}{l}0,2 \\
0,3\end{array}$ & $\begin{array}{l}2 \\
3\end{array}$ & 10,3 \\
\hline 6 & & $\begin{array}{l}0,3 \\
0,4\end{array}$ & $\begin{array}{l}3 \\
4\end{array}$ & 17,3 \\
\hline 7 & & 0,8 & 8 & 25,3 \\
\hline 8 & & $0,8 \mathrm{P}$ & 0 & 25,3 \\
\hline 9 & & $0,1 \mathrm{P}$ & 0 & 25,3 \\
\hline 10 & & $0,2 \mathrm{P}$ & 0 & 25,3 \\
\hline 11 & & $0,4 \mathrm{P}$ & 0 & 25,3 \\
\hline 12 & & $0,6 \mathrm{P}$ & 0 & 25,3 \\
\hline 13 & & $0,8 \mathrm{P}$ & 0 & 25,3 \\
\hline
\end{tabular}


Tabla 2. Pauta convencional

\begin{tabular}{|c|c|c|c|c|}
\hline SEMANA & VIAL & $\begin{array}{l}\text { VOL. } \\
\text { (mI) }\end{array}$ & $\begin{array}{l}\text { DOSIS } \\
\text { (U.B.) }\end{array}$ & $\begin{array}{c}\text { DOSIS ACUMULADA } \\
\text { (U.B.) }\end{array}$ \\
\hline 1 & \multirow[t]{4}{*}{1} & $\begin{array}{c}0,1+0,1 \mathrm{P} \\
0,4 \mathrm{P} \\
0,6 \mathrm{P} \\
0,8 \mathrm{P}\end{array}$ & 0,01 & 0,01 \\
\hline 2 & & $\begin{array}{c}0,1 \mathrm{P} \\
0,2 \\
0,4 \mathrm{P}\end{array}$ & 0,2 & 0,03 \\
\hline 3 & & $\begin{array}{c}0,4+0,2 \mathrm{P} \\
0,8 \mathrm{P}\end{array}$ & 0,4 & 0,07 \\
\hline 4 & & $\begin{array}{c}0,8 \\
0,2 \mathrm{P}\end{array}$ & 0,08 & 0,15 \\
\hline 5 & \multirow[t]{4}{*}{2} & $\begin{array}{c}0,1+0,1 \mathrm{P} \\
0,3 \mathrm{P}\end{array}$ & 0,1 & 0,25 \\
\hline 6 & & $\begin{array}{c}0,2+0,1 \mathrm{P} \\
0,4 \mathrm{P}\end{array}$ & 0,2 & 0,45 \\
\hline 7 & & $0,4+0,4 \mathrm{P}$ & 0,4 & 0,85 \\
\hline 8 & & 0,8 & 0,8 & 1,65 \\
\hline 9 & \multirow[t]{5}{*}{3} & 0,1 & 1 & 2,65 \\
\hline 10 & & 0,2 & 2 & 4,65 \\
\hline 11 & & 0,4 & 4 & 8,65 \\
\hline 12 & & 0,6 & 6 & 14,65 \\
\hline 13 & & 0,8 & 8 & 22,65 \\
\hline
\end{tabular}

del Hospital Virgen del Camino en forma de monodosis debidamente etiquetada, de forma que se garantizara la administración doble ciego de la inmunoterapia.

A lo largo de todo el estudio se realizó un registro informático de cada dosis administrada y de las reacciones adversas locales y sistémicas, que fueron clasificadas y tratadas según la normativa del Immunotherapy SubCommittee de la $E A A C T^{2}$.

Se realizó una valoración de la eficacia clínica mediante la graduación de la gravedad de la enfermedad por el médico, registro de cartilla de síntomas, consumo de medicación y PEF, y escala analógica visual. La valoración de eficacia clínica se realizó en los siguientes tiempos: T0 (antes del inicio de IT), T1 (en la $6^{\underline{a}}$ semana de tratamiento al alcanzar la dosis máxima de IT en la pauta agrupada), T1' (en la $12^{\mathrm{a}}$ semana de tratamiento al alcanzar la dosis máxima de IT en la pauta convencional) y TF (al cabo de un año de tratamiento).

Como parámetros de eficacia se incluyeron la modificación de la sensibilidad cutánea al alergeno medida mediante prick test ${ }^{21}$ y la determinación en suero de IgE, IgG e $\mathrm{IgG}_{4}$ específicas frente a $D$. pteronyssinus (Pharmacia CAP System). Las pruebas cutáneas se llevaron a cabo en 5 tiempos: P0 (antes del inicio de la IT), P6 (en la $6^{\mathrm{a}}$ semana de tratamiento al alcanzar la dosis máxima en la pauta agrupada), P12 (en la 12 semana de tratamiento al alcanzar la dosis máxima en la pauta convencional), P18 (en la $18^{\mathrm{a}}$ semana de tratamiento al administrar la $3^{\text {a }}$ dosis de mantenimiento) y P52 (al cabo de un año de tratamiento).

Por último, las determinaciones de IgE, IgG total e IgG4 específicas se realizaron en los siguientes tiempos: S0 (antes del inicio de IT), S2 (en la $2^{\text {a }}$ semana de tratamiento), S4 (en la $4^{\mathrm{a}}$ semana de tratamiento), S6 (en 
la $6^{\text {a }}$ semana de tratamiento), S8 (en la $8^{\mathrm{a}}$ semana de tratamiento), S10 (en la $10^{\circ}$ semana de tratamiento), S12 (en la 12 semana de tratamiento), S14 (en la 14 semana de tratamiento), S16 (en la 16 semana de tratamiento) y S52 (al completar un año de tratamiento).

Dieron su consentimiento para participar en el estudio 239 pacientes. De forma aleatoria se les asignó en la fase de inicio del tratamiento una pauta agrupada a 120 pacientes y una convencional a 119 pacientes. Ambos grupos fueron homogéneos en cuanto a sus características demográficas y clínicas (Tabla 3 ).

Nueve pacientes no completaron la fase de inicio y fueron excluidos del estudio (5 en el grupo de la pauta agrupada y 4 en el convencional). Uno de los pacientes del grupo de pauta agrupada hubo de ser retirado por exacerbación del asma que contraindicaba la administración de las dosis de IT en los plazos de tiempo establecidos. Otros 8 pacientes abandonaron por imposibilidad de desplazarse a la Unidad de IT para recibir las dosis correspondientes.

En la fase de mantenimiento, hubo 13 pérdidas más antes de completar el año previamente estipulado. Una paciente fue retirada del estudio por embarazo durante la fase de mantenimiento y el resto fueron abandonos, ninguno debido a reacciones adversas o agravamiento de su patología.

El tiempo necesario para alcanzar la dosis prefijada de 8 U.B. fue de 50,4 $\pm 0,97$ días $(x \pm$ ee) con la pauta agrupada y de $93,45 \pm 1,41$ días con la pauta convencional (reducción del 46\% con pauta agrupada respecto a la convencional). Se administraron 5.198 inyecciones en la fase de inicio correspondiendo a 3.544 de tratamiento activo (1.590 con pauta convencional y 1.954 con pauta agrupada) y el resto de placebo. La dosis acumulada con la pauta agrupada fue de $25,3 \pm 0,40$ U.B. y en el grupo convencional de $22,45 \pm 0,33$ U.B. En la fase de mantenimiento se administraron 2.037 dosis de tratamiento activo, hasta completar un año de tratamiento. Se produjeron reacciones adversas sistémicas todas leves, en sólo un $0,15 \%$ inyecciones de tratamiento activo, sin diferencias entre ambas pautas en cuanto a frecuencia de reacciones adversas.

Aunque la eficacia clínica de ambas pautas de inmunoterapia fue similar al año de tratamiento, la pauta agrupada indujo una disminución de los síntomas de asma (Fig. 1)y una reducción en los síntomas y el consumo de medicación para la rinitis (Fig. 2) desde que se alcanzó la dosis de mantenimiento de la inmunoterapia, 6 semanas antes que con la pauta convencional.

La pauta agrupada redujo la sensibilidad cutánea al alergeno ya desde el primer tiempo de control en la $6^{\mathbf{a}}$ semana, mientras que esto no ocurrió con la pauta convencional hasta la semana $12^{\text {a }}$. La disminución de la sensibilidad cutánea con ambas pautas fue similar en la semana $18^{\mathrm{a}}$ y al año de IT. Se observaron también diferencias en la modificación de los parámetros in vitro inducidas por las pautas agrupada y convencional en la semana $6^{\underline{a}}$. Los cambios más consistentes se obtuvieron con la IgG $_{4}$ específica frente a $D$. pteronyssinus (Fig. 3 ).

Tabla 3. Características demográficas y clínicas.

\begin{tabular}{|l|c|c|}
\hline & $\begin{array}{c}\text { Agrupada } \\
\mathbf{N}=\mathbf{1 2 0}\end{array}$ & $\begin{array}{c}\text { Convencional } \\
\text { N=119 }\end{array}$ \\
\hline Sexo (Hombre/Mujer) & $75 / 45$ & $71 / 48$ \\
\hline $\begin{array}{l}\text { Edad en años } \\
\text { (media } \pm \text { desviación estándar) }\end{array}$ & $19,34 \pm 9,8$ & $18,47 \pm 9,49$ \\
\hline Tiempo evolución en meses (mediana, RIQ) & $48(24-120)$ & $48(24-60)$ \\
\hline Asma bronquial (L/M/G) & $94(55 / 39 / 0)$ & $82(54 / 28 / 0)$ \\
\hline Rinitis (L/M/G) & $107(16 / 88 / 3)$ & $102(10 / 90 / 2)$ \\
\hline Fumadores & 13 & 22 \\
\hline
\end{tabular}

L/M/G : Leve/Moderada/Grave 


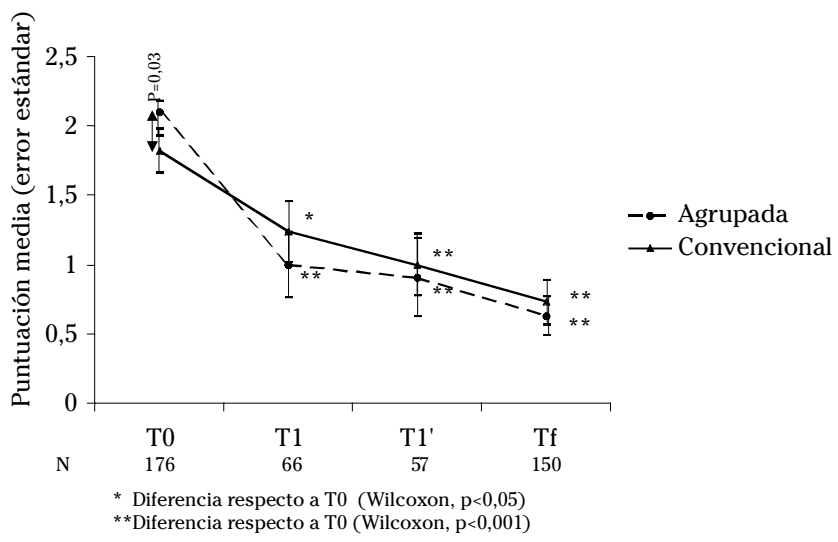

Figura 1. Puntuación de síntomas de asma.

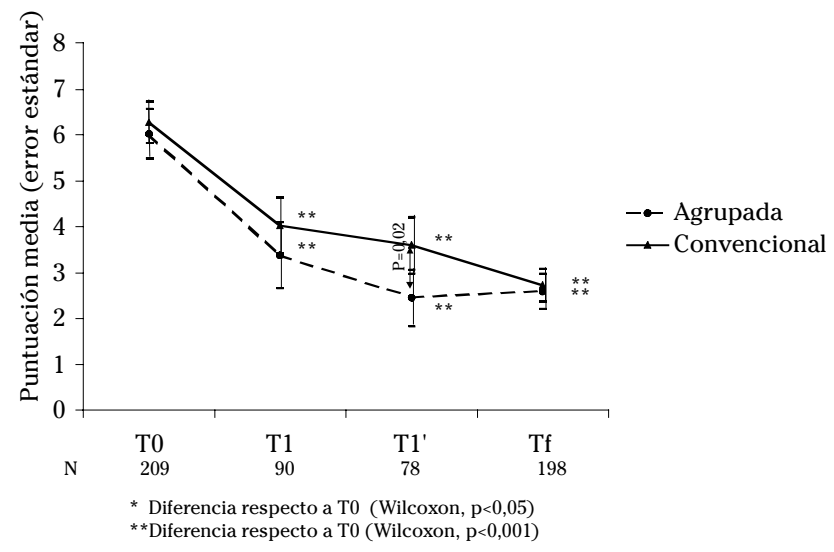

Figura 2. Puntuación de síntomas y medicación para rinitis.

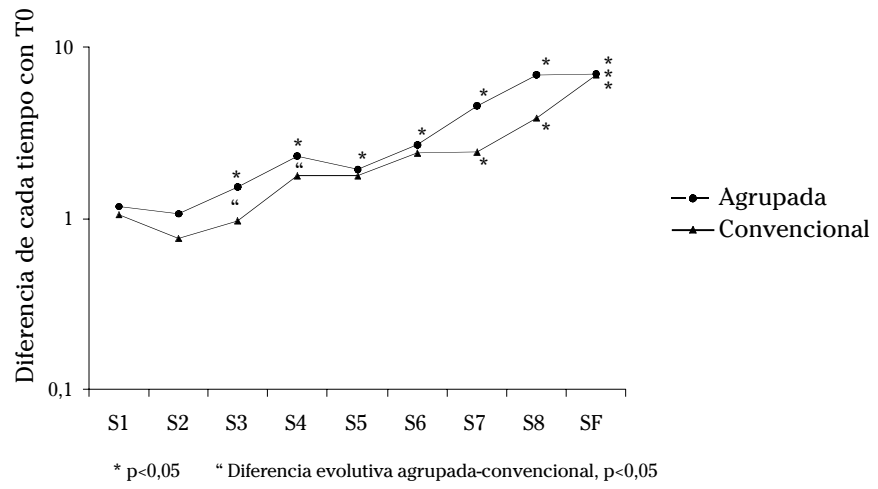

Figura 3. Evolución de $\operatorname{IgG}_{4}$ frente a $D$. pteronyssinus. 
Se detectó un aumento significativo una vez se hubo alcanzado la dosis máxima de inmunoterapia; esto ocurrió 6 semanas antes con la pauta agrupada que con la convencional. Después de un año de tratamiento los cambios en los parámetros in vitro fueron similares para ambos grupos de tratamiento.

En definitiva, la pauta agrupada ensayada reduce en un $46 \%$ el tiempo necesario para alcanzar la dosis de mantenimiento del tratamiento con la misma seguridad que la pauta convencional, y ofrece la ventaja de adelantar la eficacia clínica y la modificación de la sensibilidad cutánea al alergeno y de los parámetros in vitro, de forma paralela a la dosis de inmunoterapia administrada.

\section{BIBLIOGRAFÍA}

1. MALLING HJ. Immunotherapy as an effective tool in allergy treatment. Allergy 1998; 53 : 461-472.

2. Immunotherapy SubCommittee E.A.A.C.I Immunotherapy. Position Paper. Allergy 1993; 48: 1-45.

3. Bousouet J, LOCKEy RF, MaLling HG. WHO Position Paper. Allergen Immunotherapy: therapeutic vaccines for allergic diseases. Allergy 1998; 53: 1-42.

4. Abramson MJ, PuY R, Weiner J Immunotherapy in asthma: an updated systematic review. Allergy 1999; 54: 10221041.

5. TABar AI, García BE, Rodriguez A, Olaguibel JM, MuRo MD, Quirce S. A prospective safetymonitoring study of immunotherapy with biologically standardized extracts. Allergy 1993; 48: 450-453.

6. TABar AI, Echechipía S, García BE, Olaguibel JM, Gómez B, LASA E et al. Safety-monitoring of allergen-specific immunotherapy with standardized extracts. A prospective and predictive study in 7000 patients. Allergy 2001; 56: 91.

7. Paranos S, Petrovic S. Early effects of rush immunotherapy with Dermatophagoides pteronyssinus in asthmatics. J Invest Allergol Clin Immunol 1997; 7: 588-595.

8. Vidal C, Fernández-Távora L, Moreno C, GUARDIA P. Inmunoterapia cluster con extracto depot de Dermatophagoides pteronyssinus: estudio prospectivo de tolerancia. Rev Esp Alergol Inmunol Clin 1997; 12: 351-355.
9. Mellerup MT, Hahn GW, Poulsen LK, Malling HJ. Safety of allergen-specific immunotherapy. Relation between dosage regimen, allergen extract, disease and systemic side-effects during induction treatment. Clin Exp Allergy 2000; 30: 14231429 .

10. García-Ortega P, Merelo A, Marrugat J, RicharT C. Decrease of skin and bronchial sensitization following short-intensive scheduled immunotherapy in mite-allergic asthma. Chest 1993; 103: 183-718.

11. Moreno C, Fernández-TÁvora L, Vidal C Justicia JL, GUERRA F. Estudio prospectivo de seguridad de inmunoterapia agrupada con extractos acuosos de pólenes. Allergol Immunol Clin 2001; 16: 95-101.

12. Walker SM, Pajno GB, Torres Lima M, Wilson DR, DURHAM SR. Grass pollen immunotherapy for seasonal rhinitis and asthma: A randomized, controlled trial. J Allergy Clin Immunol 2001; 107: 87-93.

13. Muñoz D, Bernaola G, Audícana M, Fernández E, FERNÁNDEZ DE CORRES L. Inmunoterapia rápida en pacientes polínicos. Evolución clínica y variación de parámetros in vivo e in vitro. Rev Esp Alergol Inmunol Clin 1991; 6 : 57-61.

14. FERnÁNDEZ-Távora L, García D. Inmunoterapia Cluster con extractos de pólenes: estudios de tolerancia, cambios inmunológicos y eficacia clínica. Rev Esp Alergol Inmunol Clin 1991; 6: 68-74

15. Stevens WJ, Verhelst JA, VAn den Bogaert W, BRIDTS $\mathrm{CH}$. Clinical and biological evaluation of semi-rush and ordinary immunotherapy schemes in type I allergic respiratory diseases. Allergy 1985; 40: 447-452.

16. Malding HJ, Dreborg S, Weeke B. Diagnosis and immunotherapy of mould allergy. V. Clinical efficacy and side effects of immunotherapy with Cladosporium herbarum. Allergy 1986; 41: 507-519.

17. Moreno C, Fernández-Távora L, Justicia JL, Cabeza N, Vidal C. Búsqueda de una pauta idónea para iniciación de inmunoterapia con un extracto de Alternaria tenuis. Alergo Inmunol Clin 2001; 16: 133-175.

18. Fernández-Távora L, García Fernández D, GARRIDO G. Inmunoterapia cluster: la mejor alternativa para las Unidades de Inmunoterapia. Experiencia personal. Rev Esp Alergol Inmunol Clin 1994; 9: 65.

19. Moreno C, Fernández-Távora L, Justicia JL Cabeza N, Guerra F. Perfil asistencial de una pauta agrupada de inmunoterapia con extractos de pólenes absorbidos en 
hidróxido de aluminio. Alergol Inmunol Clin 2001; 16: 329-333.

20. Tabar AI, Muro MD, García BE, Alvarez MJ, ACERO S, Rico P et al. Dermatophagoides pteronyssinus cluster immunotherapy. A controlled trial of safety and clinical efficacy. Invest Allergol Clin Immunol 1999; 9: 155-164.

21. DREBorg S. Skin tests used in type 1 allergy skin testing. Position Paper of the European Academy of Allergy and Clinical Immunology. Allergy 1989: 44 (Suppl. 10) 